\title{
Effect of AR-13324 on Episcleral Venous Pressure in Dutch Belted Rabbits
}

\author{
Jeffrey W. Kiel ${ }^{1}$ and Casey C. Kopczynski ${ }^{2}$
}

\begin{abstract}
Purpose: AR-13324 is a potential new drug for the treatment of patients with glaucoma that has been shown to lower intraocular pressure (IOP) by increasing trabecular outflow facility and decreasing aqueous production. The present study tested the hypothesis that AR-13324 also lowers IOP by reducing episcleral venous pressure (EVP). Methods: In Dutch Belted (DB) rabbits $(n=11)$, arterial pressure (AP), IOP, carotid blood flow (BFcar), heart rate $(\mathrm{HR})$, and EVP were measured invasively. Animals were dosed with AR-13324 $(0.04 \%$, topical, $n=6)$ once daily for 3 days. On day 3 , the animals were anesthetized, and then, measurements were obtained before dosing with AR-13324 or vehicle $(n=5)$ and for $3 \mathrm{~h}$ after dosing. The data (mean \pm standard error of the mean) were analyzed by repeated measures ANOVA with post hoc testing. Retrospective baseline data from prior similar studies in New Zealand White rabbits were also compiled.

Results: Baseline values were as follows: AP, $101 \pm 3 \mathrm{mmHg}$; IOP; $33 \pm 3 \mathrm{mmHg}$; EVP, $16 \pm 1 \mathrm{mmHg}$; BFcar, $41 \pm 4 \mathrm{~mL} / \mathrm{min}$; and HR, $330 \pm 6 \mathrm{bpm}$. Three hours after AR-13324 dosing, IOP was reduced by $39 \% \pm 7 \%$ $(P<0.001)$ and EVP decreased by $35 \% \pm 4 \%(P<0.05)$; after vehicle dosing, IOP was reduced by $24 \% \pm 4 \%$ $(P<0.05)$ and EVP increased by $25 \% \pm 5 \%(P<0.05)$. AP, BFcar, and HR were unchanged.

Conclusions: AR-13324 produces statistically significant lowering of EVP in DB rabbits. In addition, the baseline values for AP, IOP, EVP, BFcar, and HR in the DB rabbit are higher than those previously reported in the New Zealand rabbit.
\end{abstract}

\section{Introduction}

$\mathbf{T}$ HE PRIMARY PURPOSE of this study was to test the hypothesis that AR-13324 decreases episcleral venous pressure (EVP). AR-13324 is a new compound that is in clinical development for the lowering of intraocular pressure (IOP) in patients with glaucoma and ocular hypertension. Phase 2 clinical testing found that AR-13324 decreased IOP significantly over a 28 -day period. ${ }^{1}$ AR-13324 is an amino isoquinoline amide that has been reported to have an inhibitory activity against both rho-associated protein kinase (ROCK) and norepinephrine transporter (NET) (deLong, M.A. et al. Association for Research in Vision and Ophthalmology. Invest Ophthalmol Vis Sci, 2012; 53: E-Abstract 3867 and Kopczynski, C. et al. Association for Research in Vision and Ophthalmology. Invest Ophthalmol Vis Sci, 2012; 53: E-Abstract 5080). ROCK inhibition is known to decrease actomyosin contraction in smooth muscle cells, including the smooth muscle-like cells of the trabecular meshwork. ${ }^{2}$ In nonhuman primates, AR-13324 has been shown to increase trabecular outflow facility, ${ }^{3}$ consistent with other ROCK inhibitors. ${ }^{2}$ However, unlike other ROCK inhibitors, AR-13324 also decreased the aqueous production. ${ }^{3}$ It was proposed that this second mechanism of IOP lowering may be related to the NET inhibition, which can increase adrenergic transmission by blocking the reuptake of norepinephrine at adrenergic synapses. ${ }^{4}$ Given that norepinephrine activates alpha-adrenergic receptors, a third possible mechanism by which AR-13324 could lower IOP is by a decrease in the EVP. ${ }^{5}$

The secondary purpose of this study was to determine the EVP in the Dutch Belted (DB) rabbit, an animal model used in the preclinical testing of ophthalmic drugs. ${ }^{6}$ Two advantages of the DB rabbit over the New Zealand White (NZW) rabbit, another commonly used preclinical model, are (i) the DB rabbit eye is pigmented and, so, more closely replicates the binding of drug to melanin as occurs in the human eye, ${ }^{7,8}$ and (ii) the baseline IOP in the DB rabbit is high (ie, $25-30 \mathrm{mmHg})^{9}$ relative to the $\mathrm{NZW}$ rabbit $(\approx 5 \mathrm{mmHg}),{ }^{10}$ which tends to accentuate the effect of

\footnotetext{
${ }^{1}$ Department of Ophthalmology, University of Texas Health Science Center at San Antonio, San Antonio, Texas.

${ }^{2}$ Aerie Pharmaceuticals, Inc., Research Triangle Park, North Carolina.

(C) Kiel and Kopczynski 2015; Published by Mary Ann Liebert, Inc. This Open Access article is distributed under the terms of the Creative Commons Attribution Noncommercial License (http://creativecommons.org/licenses/by-nc/4.0/) which permits any noncommercial use, distribution, and reproduction in any medium, provided the original author(s) and the source are credited.
} 
antihypertensive drugs on IOP. The cause of this relatively high baseline IOP is unclear, but it does not appear to be due to a higher aqueous production or lower outflow facility than in the NZW rabbit (C. Toris and M. McLaughlin, pers. comm.). To the best of our knowledge, uveoscleral outflow has not been measured in the DB rabbit, but in the NZW rabbit, uveoscleral outflow accounts for 5\%-50\% of total outflow, depending on the method used to calculate it. ${ }^{11,12}$ Similarly, EVP has not been measured in the DB rabbit, but it is $\sim 10 \mathrm{mmHg}$ in the NZW rabbit. ${ }^{5,13}$

\section{Methods}

The animal procedures were approved by the Institutional Animal Care and Use Committee and conducted in accordance with the ARVO Statement for the Use of Animals in Ophthalmic and Vision Research. At the end of the experiment, all animals were euthanized with an overdose of anesthetic without regaining consciousness.

\section{Animal model}

Male DB rabbits $(n=11,2.15 \pm 0.09 \mathrm{~kg}$; Millbrook Labs, Amherst, MA) were used in this study. The animals were housed in the University of Texas Health Science Center at San Antonio vivarium with food and water available ad libitum. On the experiment day, the animals were anesthetized with pentobarbital sodium $(30 \mathrm{mg} / \mathrm{kg}$, i.v., supplemented as needed). The animals were intubated through a tracheostomy and respired with room air. Expired $\mathrm{PCO}_{2}$ was monitored (SurgiVet V9004; Sims BCI, Inc., Waukesha, WI) and maintained at $\approx 40 \mathrm{mmHg}$. A heating pad was used to maintain normal body temperature $\left(38^{\circ} \mathrm{C}-39^{\circ} \mathrm{C}\right)$. All intravenous injections were given through cannulated marginal ear veins.

During the tracheostomy, the right common carotid was isolated for later placement of a transit-time ultrasound flow probe (2PSB; Transonics Systems, Ithaca, NY) to measure carotid blood flow (BFcar, TS420 flowmeter; Transonic Systems) and a cardiotachometer to measure the heart rate (HR). The right ear artery was cannulated to measure arterial pressure (AP) at eye level using a catheter connected to a pressure transducer zeroed at eye level. After the initial surgical preparation, the animals were mounted on a stereotaxic head holder and paralyzed with gallamine triethiodide ( $1 \mathrm{mg} / \mathrm{kg}$, i.v., supplemented as needed) to eliminate eye movement. Once the animal was stable, the ultrasound flow probe was placed on the carotid artery and a 23-gauge needle was inserted into the vitreous cavity of the right eye through the pars plana to measure the IOP with a second pressure transducer. To avoid the rabbit ocular trauma response and release of prostaglandins, ${ }^{14}$ the right eye was anesthetized topically with lidocaine before the cannulation and extra care was taken not to disturb the cornea and anterior chamber. After the eye cannulation, any residual lidocaine was removed with a saline rinse.

A micropipette-based servonull pressure system (Model 900A; World Precision Instruments, Sarasota, FL) was used to measure the pressure in episcleral veins. ${ }^{15}$ Borosilicate glass capillary tubes $(1.0 \mathrm{~mm}$ diameter, 1B100-6; World Precision Instruments) were used to make the micropipettes with a pipette puller (P-87; Sutter, Novato, CA). Under visual observation with a dissecting microscope (Olympus SZSTB1), the tips were triple beveled in 3 planes to achieve an inner diameter of $2-4 \mu \mathrm{m}$ (BV-10 beveller with 104-F grinding plate; Sutter). For EVP measurement, the micropipettes were filled with a $2 \mathrm{M}$ sodium chloride solution and connected to the servonull pressure system. To complete the servonull electrical circuit, the ground wire was placed within the dermal opening of the tracheostomy. The episcleral veins at the superior limbus location were targeted. When needed, a small incision $(2-3 \mathrm{~mm})$ was made in the conjunctiva to expose the episcleral vessels. A small salinefilled pool over the target vessels was used to zero the pipette tip. A surgical microscope (MC-M3101XY; World Precision Instruments) was used to position the tip in the pool and to cannulate the episcleral veins, which were identified by the streaming of aqueous in the flow of blood. The vessels were generally cannulated near their exit point from the sclera.

\section{Protocol}

The protocol entailed once per day $(\approx 8: 00 \mathrm{AM})$ - topical application of 1 drop of $0.04 \%$ AR-13324 for 2 days. Then, on the morning of the third day $(\approx 8: 00 \mathrm{AM})$, the animals were anesthetized and surgically prepared, and then, measurements were obtained before dosing with AR-13324 or vehicle (baseline measurements) and for $3 \mathrm{~h}$ after dosing. The 3-day dosing regimen was chosen based on an in-house IOP-lowering data from a related compound (AR-13165the racemate of AR-13324), which indicated that it took 3-5 days of dosing to reach peak efficacy in DB rabbits (deLong, M.A. et al. ARVO. Invest Ophthalmol Vis Sci, 2012; 53: EAbstract 3867 and Kopczynski, C. et al. ARVO. Invest Ophthalmol Vis Sci, 2012; 53: E-Abstract 5080). Consequently, 3 days of dosing with AR-13324 seemed likely to increase the probability of a robust EVP response.

\section{Drug and vehicle}

AR-13324 ophthalmic solution $0.04 \%$ was provided as a sterile borate-mannitol buffered aqueous solution with a $\mathrm{pH}$ of $\sim 5$ with $0.015 \%$ benzalkonium chloride as a preservative. The vehicle lacked the active compound, but was otherwise identical.

\section{Data analysis}

All parameters were digitally recorded (LabChart 7, v7.2.5; ADInstruments, Colorado Springs, CO) on a Mac Mini computer. The data were initially analyzed by averaging the measured variables over 5-10-min periods during baseline before drug or vehicle administration and then at hourly intervals for 3-h afterward. The averaged values were compiled in an Excel spreadsheet and analyzed with GraphPad Prism (v5.0) using repeated measures ANOVA and Bonferroni post hoc testing to identify statistically significant changes. GraphPad Prism was also used for graphic analysis. Data are presented as the mean \pm standard error of the mean. A 2-tailed $p$-value of 0.05 or less was considered statistically significant.

Also, a retrospective compilation of baseline data was performed using several EVP studies in NZW rabbits conducted by 1 of the authors (Jeffrey W. Kiel). ${ }^{5,13,15-17}$ The animal anesthesia, surgical procedures, and maintenance were the same as in the present study. Baseline values between rabbit strains were compared with an unpaired Student's $t$-test. 


\section{Results}

Figure 1 shows representative traces from an AR-13324 experiment and a vehicle experiment that illustrate the protocol.

In the AR-13324 group $(n=6)$, the baseline average values were as follows: AP, $105 \pm 3 \mathrm{mmHg}$; IOP, $34 \pm 4 \mathrm{mmHg}$; EVP, $17 \pm 2 \mathrm{mmHg} ; \quad$ BFcar, $35 \pm 1 \mathrm{~mL} / \mathrm{min}$; and HR, $337 \pm 9 \mathrm{bpm}$. Three hours after AR-13324 dosing, the IOP was reduced by $39 \% \pm 7 \%$ (to $19 \pm 2 \mathrm{mmHg}, P<0.001$ ) and the EVP by $35 \% \pm 4 \%$ (to $11 \pm 1 \mathrm{mmHg}, P<0.05$ ). AP, BFcar, and HR remained stable throughout the experiment. The results are presented graphically in Fig. 2.

In the vehicle group $(n=5)$, the baseline average values were as follows: AP, $96 \pm 3 \mathrm{mmHg}$; IOP, $32 \pm 3 \mathrm{mmHg}$; EVP, $15 \pm 1 \mathrm{mmHg}$; BFcar, $47 \pm 7 \mathrm{~mL} / \mathrm{min}$; and HR, $322 \pm 9$. Three hours after application of the vehicle, the IOP was reduced by $24 \% \pm 4 \%$ (to $25 \pm 3 \mathrm{mmHg}, P<0.05$ ) and EVP increased by $25 \% \pm 5 \%$ (to $19 \pm 1 \mathrm{mmHg}, P<0.05$ ). BP, $\mathrm{BF}$ car, and HR were unchanged. The results are presented graphically in Fig. 3.
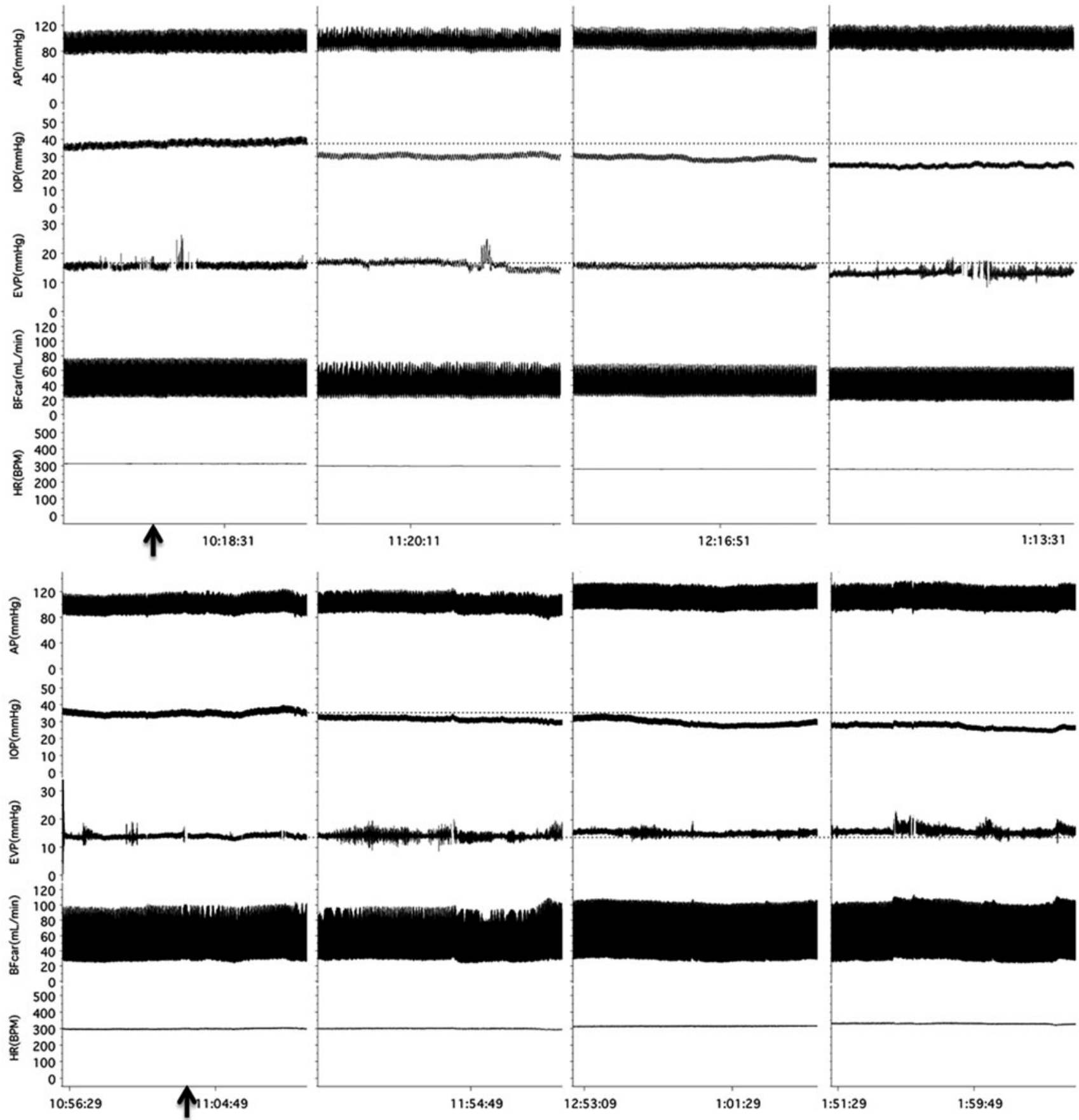

FIG. 1. Experimental tracings show the protocols for the AR-13324 (top) and vehicle (bottom) experiments; arrows indicate the application times for drug and vehicle. AP, arterial pressure; BFcar, carotid blood flow; EVP, episcleral venous pressure; HR, heart rate; IOP, intraocular pressure. 


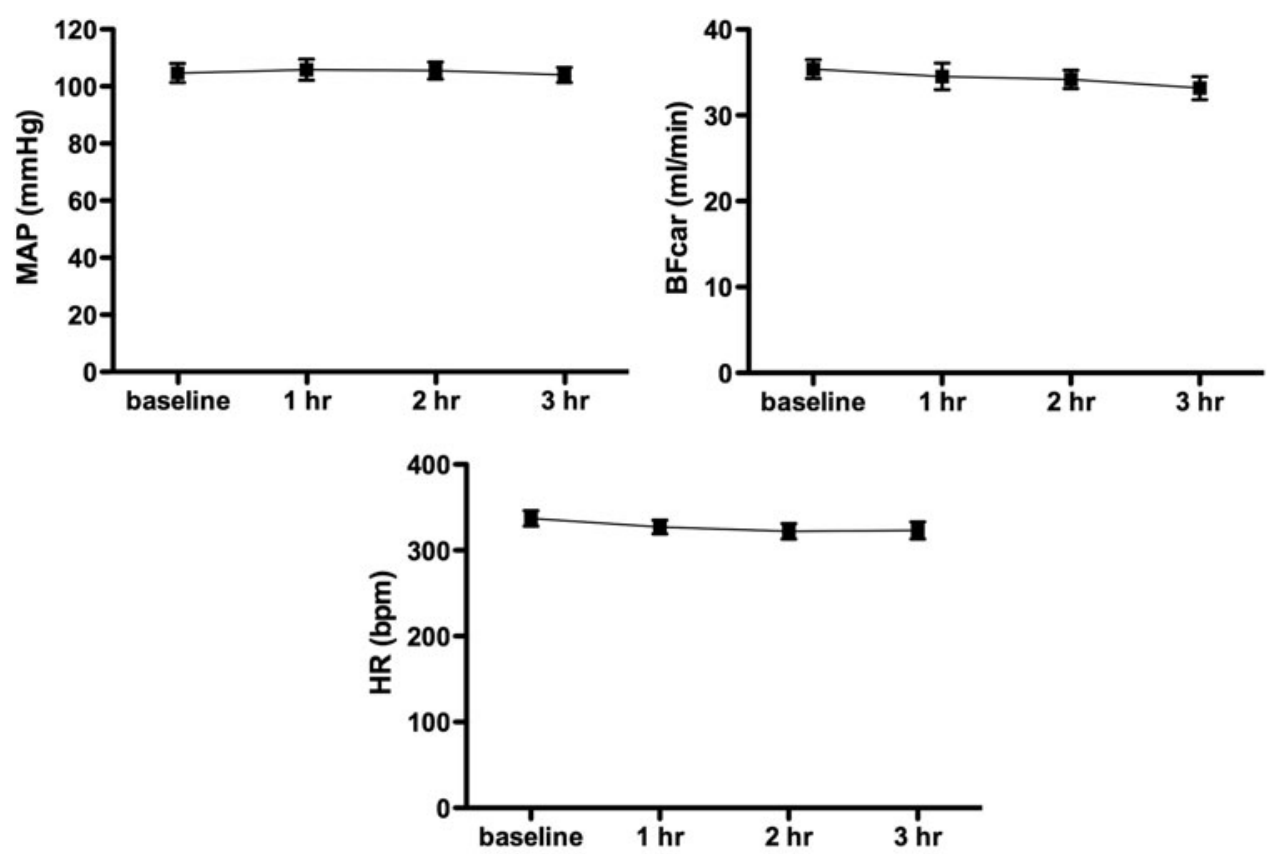

FIG. 2. Graphs of group mean responses to AR-13324 for measured variables. *Indicates significant difference from baseline. MAP, mean arterial pressure.
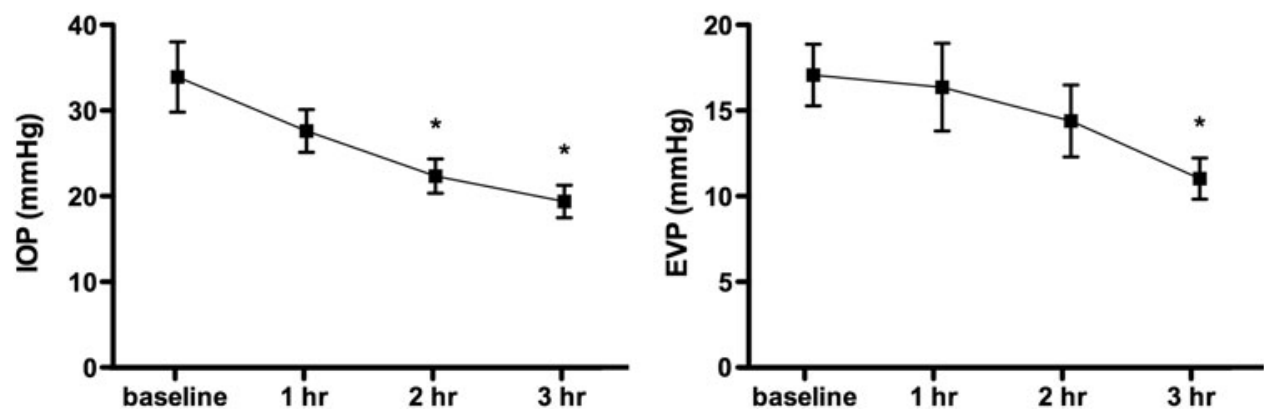

Table 1 shows the comparison of the baseline values for AP, IOP, EVP, BFcar, and HR for the DB rabbits in this study and the NZW rabbits in several published studies. All measured variables were significantly different between the rabbit strains.

\section{Discussion}

Consistent with the study hypothesis, AR-13324 lowered the EVP significantly. The vehicle results confirmed that the EVP-lowering effect was due to the active compound, rather than the vehicle. At baseline, half of the measured IOP in the AR-13324 treated group ( $34 \mathrm{mmHg}$ ) was attributable to EVP $(17 \mathrm{mmHg})$, assuming the 1:1 correspondence between IOP and EVP in the Goldmann equation. ${ }^{18}$ At $3 \mathrm{~h}$ postdosing, the reduction in EVP caused by AR-13324 treatment accounted for $42 \%$ of the measured reduction in IOP. The remaining reduction in IOP achieved by AR-13324 is presumed to occur through 1 or more additional mechanisms of action, most likely increased outflow facility and decreased aqueous production, as occurred in a previous primate study. ${ }^{3}$

AR-13324 is reported to be an inhibitor of both ROCK and NET (deLong, M.A. et al. Association for Research in Vision and Ophthalmology. Invest Ophthalmol Vis Sci, 2012; 53: EAbstract 3867 and Kopczynski, C. et al. Association for Research in Vision and Ophthalmology. Invest Ophthalmol Vis Sci, 2012; 53: E-Abstract 5080). There is convincing evidence that ROCK inhibitors elicit vasodilation, ${ }^{19}$ whereas
NET inhibitors are potential vasoconstrictors. ${ }^{4}$ It is not particularly clear which effect should predominate in the episcleral circulation and drive EVP, since the interplay of the blood flow and vascular resistances in the episcleral supply arterioles, the arteriovenous anastomoses, and muscular veins is complex. ${ }^{15,20-22}$ Brimonidine, a known vasoconstrictor, has been shown to decrease EVP in rabbits, ${ }^{5}$ and nitroprusside, a known vasodilator, has been shown to increase EVP in rabbits. $^{5,13}$ So, the decrease in EVP in response to AR-13324 seems likely to be mediated, at least in part, by its NET inhibitory action. Additional studies using a more selective ROCK inhibitor will be needed to determine if ROCK inhibition alone is able to reduce the EVP.

An unexpected finding in the vehicle-treated animals is that IOP declined at 2 and $3 \mathrm{~h}$ after topical dosing. It is unclear whether this was an effect of the vehicle, the amount of time under anesthesia, or perhaps the small group size. The simple composition of the vehicle makes it unlikely that it would decrease aqueous production, increase outflow facility, or increase uveoscleral outflow, and so account for the decrease in IOP. Also, any corneal or conjunctival irritation would likely elicit the rabbit ocular injury response and a rise in IOP. ${ }^{14}$ Thus, it seems unlikely that the vehicle was responsible for the IOP reduction observed in the vehicle-treated animals, although this cannot be ruled out completely.

Another noteworthy finding of this study was that the baseline values for AP, IOP, EVP, BFcar, and HR in the DB rabbit were markedly higher than for the NZW rabbit 
FIG. 3. Graphs of control group mean responses to vehicle for measured variables. *Indicates significant difference from baseline.
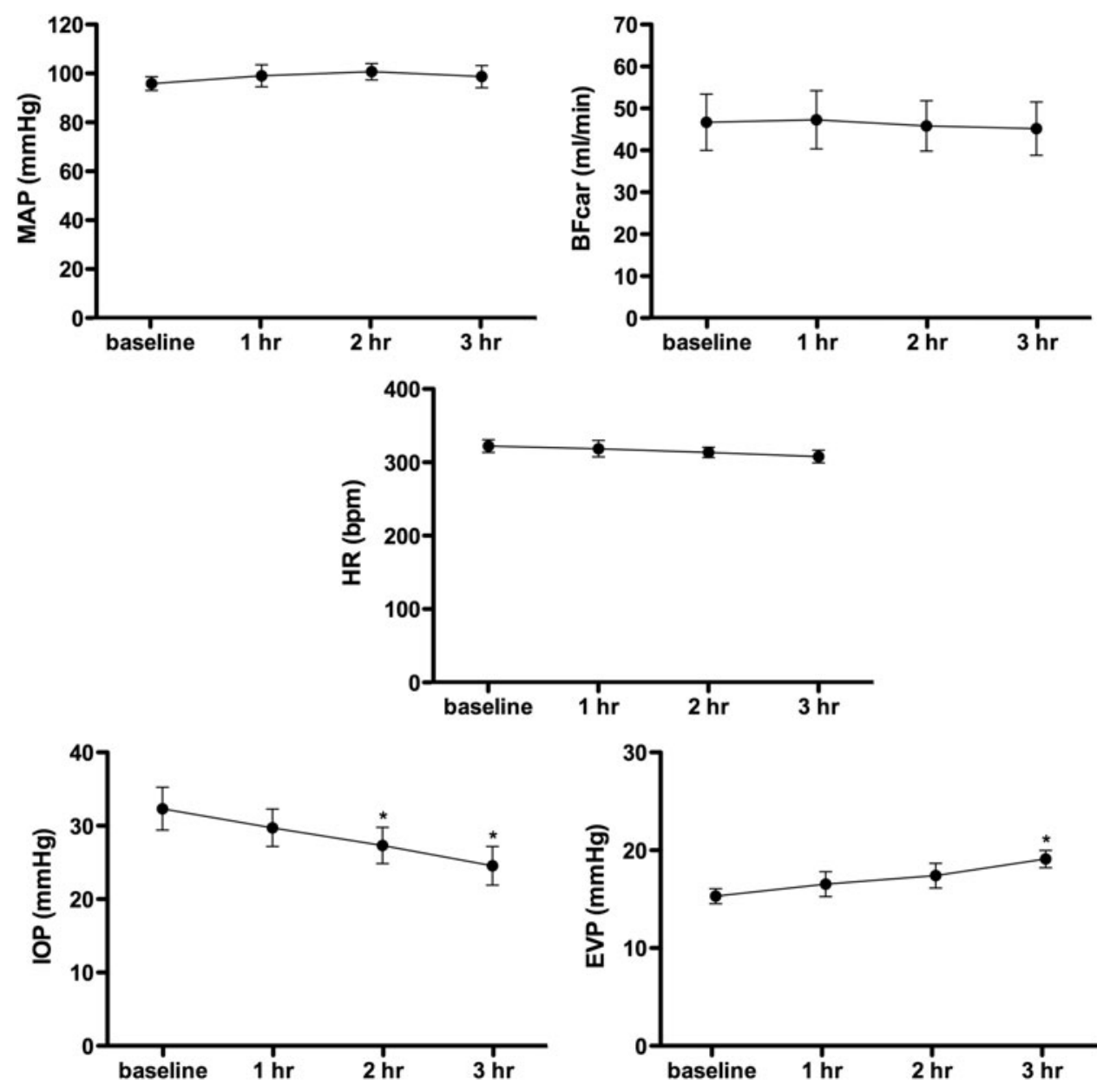

under similar experimental conditions. In the DB rabbit, EVP accounted for $49 \%$ of the baseline IOP, whereas it accounted for $64 \%$ in the NZW, so EVP is clearly an important facet of aqueous dynamics in both strains. Another interesting finding is that the IOP in the DB rabbit was high

Table 1. Baseline Comparison for Dutch Belted and New Zealand White Rabbits

\begin{tabular}{lccccc}
\hline Strain & $\begin{array}{c}\text { MAP } \\
(m m H g)\end{array}$ & $\begin{array}{c}\text { IOP } \\
(\mathrm{mmHg})\end{array}$ & $\begin{array}{c}\mathrm{EVP} \\
(\mathrm{mmHg})\end{array}$ & $\begin{array}{c}\text { BFcar } \\
(\mathrm{mL} / \mathrm{min})\end{array}$ & $\begin{array}{c}H R \\
(\mathrm{bpm})\end{array}$ \\
\hline DB & & & & & \\
Mean & 100.7 & 33.2 & 16.3 & 41.0 & 330.3 \\
SD & 8.4 & 8.3 & 3.4 & 11.7 & 20.7 \\
SE & 2.5 & 2.5 & 1.0 & 3.7 & 6.3 \\
$n$ & 11 & 11 & 11 & 10 & 11 \\
NZW & & & & & \\
Mean & 70.4 & 16.2 & 10.4 & 28.3 & 291.2 \\
SD & 6.1 & 4.5 & 2.5 & 8.9 & 27.8 \\
SE & 0.6 & 0.5 & 0.3 & 1.0 & 3.0 \\
$n$ & 99 & 97 & 98 & 85 & 87 \\
$P$-value & $<0.001$ & $<0.001$ & $<0.001$ & $<0.001$ & $<0.001$ \\
\hline
\end{tabular}

BFcar, carotid blood flow; DB, Dutch Belted; EVP, episcleral venous pressure; HR, heart rate; IOP, intraocular pressure; MAP, mean arterial pressure; $n$, number of animals; NZW, New Zealand White; SD, standard deviation; SE, standard error of the mean. enough that the strain should be prone to glaucoma, but we found no evidence of this in the literature. One possible explanation is that the arterial pressure in the DB rabbit is higher than in the NZW rabbit (also not prone to glaucoma), such that the perfusion pressure in the DB rabbit is actually greater than in the NZW rabbit $(67 \mathrm{mmHg}$ vs. $54 \mathrm{mmHg}$ ), which may be protective.

In conclusion, the results indicate that AR-13324 decreases EVP in the DB rabbit. If it is found to have a similar effect in humans, it would offer an important new mechanism of action for lowering IOP in patients with glaucoma.

\section{Acknowledgments}

The support of the van Heuven endowment is sincerely appreciated. This project was funded by a grant from Aerie Pharmaceuticals, Inc. to the University of Texas Health Science Center at San Antonio.

\section{Author Disclosure Statement}

J.W.K. is a member of the faculty of the University of Texas Health Science Center at San Antonio. He performed the experiments. He has no proprietary interest in AR-13324 and no financial relationship with Aerie Pharmaceuticals, Inc. C.C.K. is an employee of Aerie Pharmaceuticals, Inc. and assisted in the development of the experimental design as well as the data analysis and interpretation. 


\section{References}

1. Bacharach, J., Dubiner, H.B., Levy, B., Kopczynski, C.C., and Novack, G.D. Double-masked, randomized, dose-response study of AR-13324 versus latanoprost in patients with elevated intraocular pressure. Ophthalmology. 122:302-307, 2014.

2. Inoue, T., and Tanihara, H. Rho-associated kinase inhibitors: a novel glaucoma therapy. Prog. Retin. Eye Res. 37:112, 2013.

3. Wang, R.-F., Williamson, J.E., Kopczynski, C., and Serle, J.B. Effect of 0.04\% AR-13324, a ROCK, and norepinephrine transporter inhibitor, on aqueous humor dynamics in normotensive monkey eyes. J. Glaucoma. 24:51-54, 2015.

4. Bonisch, H., and Bruss, M. The norepinephrine transporter in physiology and disease. Handb. Exp. Pharmacol. 175: 485-524, 2006.

5. Reitsamer, H.A., Posey, M., and Kiel, J.W. Effects of a topical alpha2 adrenergic agonist on ciliary blood flow and aqueous production in rabbits. Exp. Eye Res. 82:405-415, 2006.

6. Prasanna, G., Carreiro, S., Anderson, S., Gukasyan, H., Sartnurak, S., Younis, H., Gale, D., Xiang, C., Wells, P., Dinh, D., Almaden, C., Fortner, J., Toris, C., Niesman, M., Lafontaine, J., and Krauss, A. Effect of PF-04217329 a prodrug of a selective prostaglandin $\mathrm{EP}(2)$ agonist on intraocular pressure in preclinical models of glaucoma. Exp. Eye Res. 93:256-264, 2011.

7. Spence $S$. The Dutch-Belted rabbit: an alternative breed for developmental toxicity testing. Birth Defects Res. B Dev. Reprod. Toxicol. 68:439-448, 2003.

8. Sasaki, H., Yamamura, K., Nishida, K., Nakamura, J., and Ichikawa, M. Delivery of drugs to the eye by topical application. Prog. Retin. Eye Res. 15:583-620, 1996.

9. Tingey, D.P., Schroeder, A., Epstein, M.P., and Epstein, D.L. Effects of topical ethacrynic acid adducts on intraocular pressure in rabbits and monkeys. Arch. Ophthalmol. 110:699-702, 1992.

10. Kiel, J.W. Modulation of choroidal autoregulation in the rabbit. Exp. Eye Res. 69:413-429, 1999.

11. Nilsson, S.F. The uveoscleral outflow routes. Eye (Lond.). 11(Pt 2):149-154, 1997.

12. Zhao, M., Hejkal, J.J., Camras, C.B., and Toris, C.B. Aqueous humor dynamics during the day and night in juvenile and adult rabbits. Invest. Ophthalmol. Vis. Sci. 51:3145-3151, 2010.
13. Zamora, D., and Kiel, J. Episcleral venous pressure responses to topical nitroprusside and N-nitro-L-arginine methyl ester. Invest. Ophthalmol. Vis. Sci. 51:1614-1620, 2010.

14. Sears, M.L. Miosis and intraocular pressure changes during manometry. Arch. Ophthalmol. 63:159-166, 1960.

15. Zamora, D.O., and Kiel, J.W. Topical proparacaine and episcleral venous pressure in the rabbit. Invest. Ophthalmol. Vis. Sci. 50:2949-2952, 2009.

16. Reitsamer, H.A., and Kiel, J.W. A rabbit model to study orbital venous pressure, intraocular pressure, and ocular hemodynamics simultaneously. Invest. Ophthalmol. Vis. Sci. 43:3728-3734, 2002.

17. Lavery, W.J., and Kiel, J.W. Effects of head down tilt on episcleral venous pressure in a rabbit model. Exp. Eye Res. 111:88-94, 2013.

18. Goldmann, H. Abflussdruck, minutenvolumen und widerstand der kammerwasser-stromung des menschen. Doc. Ophthalmol. 5-6:278-356, 1951.

19. Hein, T.W., Rosa Jr., R.H., Yuan, Z., Roberts, E., and Kuo, L. Divergent roles of nitric oxide and rho kinase in vasomotor regulation of human retinal arterioles. Invest. Ophthalmol. Vis. Sci. 51:1583-1590, 2010.

20. Rohen, J., and Funk, R. Vasculature of the anterior eye segment. Prog. Retin. Eye Res. 13:653-685, 1994.

21. Rohen, J.W., and Funk, R. Functional morphology of the episcleral vasculature in rabbits and dogs: presence of arteriovenous anastomoses. J. Glaucoma. 3:51-57, 1994.

22. Selbach, J.M., Schonfelder, U., and Funk, R.H. Arteriovenous anastomomoses of the episcleral vasculature in the rabbit and rat eye. J. Glaucoma. 7:50-57, 1998.

Received: November 17, 2014 Accepted: February 2, 2015

Address correspondence to: Dr. Jeffrey W. Kiel Department of Ophthalmology

University of Texas Health Science Center at San Antonio MC 6230

7703 Floyd Curl Drive San Antonio, TX 78229-3900

E-mail:kiel@uthscsa.edu 